\title{
Flexibilidad laboral de la población ocupada: un análisis espacial en México, 2005 y 2014
}

\author{
Labor Flexibility of the Working Population: \\ a Spatial Analysis in Mexico, 2005 and 2014
}

\section{A flexibilidade laboral da população ativa: uma análise espacial no México, de 2005 e 2014}

\section{Yuliana Gabriela Román Sánchez ${ }^{1}$}

Profesora investigadora del Centro de Investigación y Estudios Avanzados de la Población (CIAEP) de la Universidad Autónoma del Estado de México, Toluca, México madon_dl@hotmail.com; ygromans@uaemex.mx

\section{Wendy Ovando Aldana ${ }^{2}$}

Profesora de tiempo completo de la Facultad de Economía de la Universidad Autónoma del Estado de México, Toluca, México wovandoa@uaemex.mx 


\title{
Resumen
}

El presente artículo tiene como objetivo analizar las diferencias regionales de las condiciones de trabajo que tiene la población ocupada en México. Para ello, se estima un índice de flexibilidad laboral considerando los años 2005 y 2014. El estudio se sustenta en la vertiente teórica denominada segmentación de mercados de trabajo. Los resultados muestran la existencia de diferencias regionales en el territorio mexicano que permiten identificar un patrón espacial visualizado en 2005 y 2014. Las entidades del norte de México muestran un índice de flexibilidad bajo, las zonas del centro un índice medio y los estados del sur un índice de flexibilidad alto. Lo anterior confirma que los mercados de trabajo en México ostentan la flexibilidad como particularidad trascendental, fenómeno observado en la actualidad y antes de la reforma laboral.

Palabras clave: mercado de trabajo; índice de flexibilidad laboral; diferencias regionales.

Clasificación JEL: J21, C43, R12

\begin{abstract}
This article aims to analyze regional differences in working conditions of the working population in Mexico. To do this, an index of labor flexibility is estimated considering the years 2005 and 2014. The study is based on the Labor Market Segmentation Theory. The results show the existence of regional differences in Mexican territory which enable to identify a spatial pattern that is displayed in 2005 and 2014: The northern states of Mexico exhibit a low flexibility index, those of center of Mexico show an average index, and the southern states display a high flexibility index. This confirms that in fact, labor markets in Mexico hold flexibility as transcendental particularity, phenomenon observed before the labor reform and today.
\end{abstract}

Keywords: labor market; labor flexibility index; regional differences.

\section{Resumo}

Este artigo tem por objetivo analisar as diferenças regionais nas condições de trabalho da população ativa no México. Para fazer isso se estima um índice de flexibilidade do trabalho considerando os anos de 2005 e 2014. O estudo baseia-se na teoria de segmentação dos mercados de trabalho. Os resultados mostram a existência de diferenças regionais no território mexicano que identificam um padrão espacial em 2005 e 2014. Os estados do norte do México mostram uma flexibilidade baixa, as áreas centrais uma flexibilidade média e os estados do sul alta flexibilidade. Isto confirma que os mercados de trabalho no México ostentam a flexibilidade como particularidade transcendental, fenômeno observado na atualidade e antes da reforma trabalhista.

Palavras-chave: mercado de trabalho; flexibilidade do trabalho índice; diferenças regionais.

Este trabajo está bajo la licencia Creative Commons Attribution 3.0

¿Cómo citar este artículo? / How to quote this article?

Román-Sánchez, Yuliana y Wendy Ovando-Aldana. «Flexibilidad laboral de la población ocupada: un análisis espacial en México, 2005 y 2014 ». Sociedad y economía, No. 31 (Julio - Diciembre 2016): 193-213. 


\section{Introducción}

Durante los años de 1970, la economía mundial atravesó por un desequilibrio económico motivado por diversos factores: la concentración de capitales, la fusión de empresas monopólicas y oligopólicas, el aumento de privatizaciones, la flexibilización del proceso productivo, la crisis del estado de bienestar (welfare state) y el agotamiento del patrón de acumulación de producción rígido (Harvey 1998; Antunes 2005).

El principal efecto de esta crisis fue la disminución del beneficio (Rodríguez et al. 2015); por tanto, para poder recuperarlo, los países capitalistas adoptaron el modelo etiquetado como neoliberalismo económico, incluida su lógica de mercado y la apertura comercial como el eje rector de las actividades económicas. Sin embargo, en las empresas la forma más común para reducir los costos de producción se materializó a través de la disminución del pago al trabajador.

A raíz de la crisis económica y la búsqueda por recuperar los beneficios empresariales, algunos gobiernos y empresarios del mundo han tratado de recortar los costos mediante reformas laborales que buscan la flexibilización de la mano de obra. Destacan los gobiernos de países europeos como Alemania, Italia y España (Ferreiro 2003; Tompson 2009). Dentro de los países latinoamericanos sobresalen los gobiernos de Argentina, Perú, Ecuador y México, por mencionar algunos que han hecho modificaciones a la legislación (Vega 2005). Así, la relación capital-trabajo del keynesianismo se transformó para adoptar formas flexibles (Rodríguez et al. 2015) cuyos patrones se observan, esencialmente, en la desaparición de los contratos por tiempo indefinido, la disminución de prestaciones laborales, y el quebranto de la seguridad social y la antigüedad laboral, entre otras. Paralelamente, flexibilizar las condiciones de trabajo implicó empleo de medio tiempo, temporal, estacional, casual, o el autoempleo.

Las diferentes formas de flexibilidad laboral que se han adoptado difieren en cada espacio que se analice. Por ejemplo, en los países desarrollados surge el interés por examinar, principalmente, los trabajos de tiempo parcial y los trabajos temporales porque son éstos los que han aumentado (D'Addio and Rosholm 2005; Reynolds and Wenger 2010); mientras que en los países en desarrollo, como América Latina, las preocupaciones se concentran en temas como la informalidad, la precariedad laboral y el trabajo femenino (Infante y Klein 1991; Gutiérrez 1999; García 2001; De Oliveira 2006; Mora 2010).

Este documento tiene como premisa que si existen diferencias en las formas de flexibilidad laboral entre países, entonces ocurre lo mismo dentro de éstos. Dado este planteamiento, el artículo pretende diferenciar el grado de flexibilidad que existe dentro del territorio mexicano. De tal manera que el objetivo consiste en analizar las diferencias regionales a partir de las condiciones de trabajo que tiene la población ocupada en México, mediante la estimación de un índice de flexibilidad laboral, a escala estatal, para dos años: 2005 y 2014.

Para alcanzar el objetivo planteado, se retoma a la teoría de la segmentación de mercados de trabajo (TSMT) que establece la existencia de más de un mercado. La división del mercado de trabajo provoca a su vez el surgimiento de formas particulares de empleo (FPE): a tiempo parcial, temporal, subcontratación, etcétera (Neffa 2008), que se identifican como formas de trabajo flexibles. 
La importancia de este documento radica en la generación de un índice que permita tener un panorama regional sobre el grado de flexibilidad laboral. De manera concreta se pretende identificar las diferencias regionales en cuanto a flexibilidad laboral. Lo anterior, con la finalidad de evidenciar la necesidad de políticas laborales regionales que permitan disminuir las diferencias entre las entidades mexicanas. No obstante, una de las limitantes de esta investigación es su carácter de diagnóstico, por lo que las conclusiones a las que se llega están en función de lo que se describe a partir de un índice.

El artículo está divido en cinco secciones. La primera aborda los antecedentes del proceso de flexibilización laboral. La segunda describe el sustento teórico a la propuesta del índice de flexibilidad laboral. En la tercera se establece la metodología para estimar y estratificar dicha metodología e identificar diferentes grados. En la cuarta sección se discuten los resultados, y en la quinta se presentan las conclusiones.

\section{Proceso de flexibilización laboral}

Durante los últimos años del siglo XX, algunos países desarrollados llevaron a cabo una serie de medidas para reorganizar el sistema capitalista. Entre las estrategias destacan la reducción del gasto gubernamental, el aumento de las tasas de interés, la disminución de impuestos sobre ingresos altos, el recorte de gastos sociales, la contracción de la emisión monetaria, entre otras (García 2001), a lo cual se denomina reestructuración económica.

La reestructuración económica era necesaria y se hizo evidente mediante la disminución del estado de bienestar, característico de las prácticas keynesianas. En el caso de las economías en desarrollo, el Consenso de Washington ${ }^{3}$ daba la pauta para llevar a cabo dicha reestructuración. El objetivo principal era mantener en equilibrio las variables macroeconómicas y ceder lugar al mercado como mecanismo de asignación de recursos (Harvey 1998; García et al. 2009; Rodríguez et al. 2015). De manera paralela se inició un proceso de reestructuración productiva que implicaba cambios, principalmente, en los modelos de producción que pasaban de rígidos a flexibles y, sobre todo, en el nexo con los trabajadores (De la Garza 2006). Al mismo tiempo, las empresas buscaron la forma de aminorar el declive del beneficio y la productividad: la disminución de los costos, principalmente salariales (Harvey 1998; Antunes 2005; Rodríguez et al. 2015).

Con la idea de generar más empleos y promover el crecimiento económico, gobiernos y empleadores comenzaron a fomentar la flexibilización de la mano de obra, aspecto que resultaba necesario para ir acorde con el avance de los sistemas de producción flexibles de las industrias. Alemania, por ejemplo, fomentó una reforma laboral durante el período 2002-2005 en el que se redujeron los beneficios a los trabajadores y se incrementaron los incentivos para que los desempleados encontraran trabajo en un tiempo más corto. En cuanto a Italia,

3 Las diez reformas de política económica consideradas en Washington fueron: disciplina fiscal, reordenación de las prioridades del gasto público, reforma tributaria, liberalización de las tasas de interés, tipo de cambio competitivo, liberalización del comercio, liberalización de la inversión extranjera directa, privatización, desregulación y derechos de propiedad (Williamson 2003, $10-11)$. 
la reforma de 2003 "Biagi law" tenía como principal preocupación el desempleo, pero específicamente en la región sur. Las nuevas medidas permitían que las agencias de empleo privadas pudieran competir, sin ninguna restricción, con las agencias públicas en diversos servicios, además de mejorar las condiciones en las que las empresas hicieran uso del trabajo de tiempo parcial y de otras formas de empleo no estándares (trabajo por turnos, trabajo compartido, entre otros) (Tompson 2009).

De las reformas laborales europeas, destaca la española: desde los años de 1980, para disminuir el desempleo se promovieron los contratos temporales (Ferreiro 2003; Tompson 2009). Sin embargo, esta forma de contratación incrementó las diferencias salariales entre los trabajadores: "la ganancia media de un trabajador temporal" respecto de la ganancia de un trabajador indefinido disminuyó al pasar de 57,7\% en 1988 a 44,8\% en 1995 (Ferreiro 2003, 24).

Después de la reforma de 1984, España ha realizado seis reformas más hasta 2012 (1994, 1997, 2001, 2006, 2010 y 2012) (Ferreiro 2003; Tompson 2009). Todos los cambios a la legislación laboral, con el objetivo de disminuir el desempleo mediante la flexibilización laboral, no han tenido el efecto deseado, ya que éste persiste.

La situación, sobre las reformas laborales, no es diferente para los países de América Latina. La mayoría de los países de la región -a excepción de Cuba, Costa Rica, Honduras y Uruguay- han hecho modificaciones a la legislación cuyos temas de flexibilización laboral más frecuentes son modalidades de contrato, causas de despido, indemnización -cuando se finalice la relación laboral- y los aspectos salariales y jornada laboral, pero en menor grado (Vega 2005). De manera general, se puede establecer una clasificación de los países latinoamericanos que llevaron a cabo reformas laborales durante el período 1990-2005: a) los que realizaron reformas extensas con una marcada flexibilización, como Argentina y Perú; b) los que realizaron reformas menores, pero con una clara visión de la flexibilización laboral como Brasil, Colombia, Ecuador y Panamá; y c) los que efectuaron reformas al mercado laboral con un tinte de flexibilización mucho menor como Chile, Guatemala, Nicaragua, El Salvador y Paraguay (Vega 2005).

Algunos otros países, como es el caso de México, efectuaron su reforma laboral en un momento posterior a 2005. La reforma laboral mexicana fue aprobada en 2012 (DOF -Diario Oficial de la Federación- 2012), pero con características propias de la flexibilidad laboral, ya que los cambios en la legislación reducen la estabilidad en el empleo, se legaliza la subcontratación y se abarata el despido, por mencionar tan solo algunos (Lóyzaga 2013). En palabras de Vega (2005), los cambios a las diferentes reformas laborales latinoamericanas generaron empleo, pero en condiciones de precariedad, y los trabajadores obtuvieron menos beneficios laborales.

De las experiencias de Europa y América Latina, se puede decir que las reformas laborales se han llevado a cabo con el objetivo de disminuir el desempleo -ya estructural- que añaden formas flexibles del trabajo, pero que no han alcanzado su objetivo. En el 2014, el desempleo siguió siendo un asunto de preocupación. Grecia, por ejemplo, presentó la tasa más elevada con $26 \%$; le siguió España con $24 \%$ y países como Croacia y Chipre con tasas mayores a $15 \%$ o bien Portugal, Eslovaquia e Italia con una tasa mayor a 10\%, empero inferior a $15 \%$ (European 
Commission 2015). En Latinoamérica la situación no difiere en demasía. El desempleo no ha aminorado en Argentina y Perú, por ejemplo, o en Ecuador, donde el contrato por horas ha aumentado las diferencias salariales respecto de sus pares con contrato indefinido (Vega 2005).

No obstante, la semejanza entre los países desarrollados y los emergentes se presenta con una tasa de desempleo alta, aunque es diferenciable la forma en la que se aplica la flexibilidad laboral. Mientras que en los primeros es más frecuente hallar estudios sobre la situación de los trabajos de tiempo parcial y temporal (D'Addio y Rosholm 2005; Reynolds y Wenger 2010); en los segundos, las cuestiones de informalidad, terciarización, trabajo femenino y juvenil, así como la precariedad y flexibilidad laboral son los temas más comunes (Infante y Klein 1991; Gutiérrez 1999; García 2001; De Oliveira 2006).

Lo que se puede establecer es que el sistema capitalista ha impulsado la flexibilización laboral (Rodríguez et al. 2015), pero cada espacio presenta características diferentes respecto de la flexibilidad laboral; y si esto se observa entre países, lo mismo se puede esperar dentro de éstos. En otras palabras, las formas de trabajo flexible son diferentes en el espacio. A partir de lo anterior surgen algunas preguntas: ¿Cómo establecer esas desigualdades?, ¿qué mecanismo permitirá distinguir entre un grado mayor o menor de flexibilidad laboral? Hasta este momento existe poca evidencia empírica que permita llenar los interrogantes anteriormente planteados, de ahí la importancia de este documento. Para responder a dichas preguntas, el presente artículo propone estimar un índice de flexibilidad laboral, por lo que es preciso establecer la unidad de estudio que permita realizar un indicador capaz de distinguir diferentes grados de flexibilidad laboral.

El mercado de trabajo en México presenta características idóneas para poder llevar a cabo un índice de flexibilidad laboral estatal y de esta forma analizar las diferencias regionales en dos momentos del tiempo. Lo anterior se debe a que la república mexicana presenta rasgos similares a lo mostrado en el ámbito internacional. Durante el período 1983-1998 se incrementó la polarización laboral: existe un número cada vez más reducido de trabajos en mejores condiciones (remuneración, prestaciones, competitividad laboral, contratos fijos, etc.) que aquellos en los que el número de mexicanos se incrementa con los trabajos menos atractivos (menor remuneración, escasas o nulas prestaciones, precariedad laboral, contratos temporales, etc.) (Gutiérrez 1999).

Para lograr dicho objetivo es importante señalar que esta investigación toma como base teórica a la teoría de la segmentación de mercados de trabajo que se menciona a continuación.

\section{Teoría de la segmentación de mercados de trabajo (TSMT). Marco teórico para analizar la flexibilidad laboral}

La TSMT explica que el mercado de trabajo se encuentra segmentado, y debido a esta fragmentación es que se pueden justificar algunos de los fenómenos que deja de lado la teoría neoclásica como: el desempleo, la discriminación, las relaciones sociales, entre otras (Gittleman and Howell 1995). 
Con base en la segmentación se identifican dos grandes divisiones del mercado de trabajo: una donde se ubican los buenos trabajos (estables, mejores salarios, etc.), y otra, con los trabajos poco atractivos (trabajo inseguro, bajo nivel de ingreso, etc.) (Reich, Gordon, and Edwards 1973; Piore 1978; Gutiérrez 1999; Neffa 2008). Sin embargo, es importante señalar que dentro de los segmentaristas se pueden ubicar diferentes versiones: dos segmentos o mercados duales (Piore 1978); mercado de tres segmentos (Reich, Gordon and Edwards 1973), o más segmentos (Gittleman and Howell 1995).

A pesar de la existencia de diversas posturas sobre el número de segmentos, la TSMT reconoce como resultado de la división del mercado de trabajo el surgimiento de formas particulares de empleo (FPE) (Neffa 2008) o bien, las nuevas formas de trabajo flexible (Rodríguez et al. 2015), debido a que las empresas tratan de reducir los costos de la rigidez laboral mediante las FPE (Neffa 2008).

Las formas de trabajo flexible están representadas por trabajo de medio tiempo, temporal, estacional, casual, trabajadores independientes, etcétera (Edwards 2006). No obstante, estos modos de trabajo se pueden clasificar en diferentes tipos de flexibilidad: numérica, externa o cuantitativa; funcional, interna o cualitativa, y salarial. El primero está relacionado con cambios en los horarios y las tareas, movilidad espacial, horas extras y trabajo de tiempo parcial. El segundo, con las contrataciones temporales y subcontrataciones, externalización de tareas de producción, etc. El tercero, con la adaptación del salario en función de la productividad, con una propensión a la disminución (Rodríguez et al. 2015). Los autores Palacio y Álvarez (2004) consideran un tipo de flexibilidad que denominan extrema, la cual se relaciona con el empleo de migrantes y la economía sumergida.

A pesar de los avances que realiza la TSMT respecto de la teoría clásica en el sentido de considerar elementos institucionales en los mercados de trabajo, el espacio se reduce a un contexto económico (Peck 1996). El estudio del mercado de trabajo estatal no es considerado dentro de la TSMT, debido a que el análisis desde esta perspectiva se realiza en la esfera macroeconómica. Por otro lado, si se considera que la diferencia de los mercados persiste entre países, entonces las desigualdades se podrán observar en su interior, es decir, a escala estatal, municipal e incluso local.

De acuerdo con Peck (1996), la formación del mercado de trabajo y su operación es diferente en cada espacio de análisis, por lo que estudiar la dimensión espacial permitiría desarrollar los cimientos de una nueva generación de la TSMT. En la revisión de literatura se detectaron disimilitudes en los temas de estudio entre los países desarrollados y emergentes, pero resulta necesario investigar dentro de alguna nación la existencia de asimetrías; en este caso se ha elegido a México. Por ello, el presente artículo establece la siguiente hipótesis de investigación: el índice de flexibilidad laboral arrojará diferencias espaciales. Para poder corroborar esta hipótesis y alcanzar el objetivo de este estudio se describe la metodología en el siguiente apartado. 


\section{Indice de flexibilidad laboral. Estrategia metodológica}

\subsection{Fuente de datos}

La presente investigación utilizó datos obtenidos de la Encuesta Nacional de Ocupación y Empleo (ENOE) que levanta el Instituto Nacional de Estadística y Geografía (INEGI) de forma trimestral. El objetivo general de la ENOE es obtener información estadística sobre las características ocupacionales de la población mexicana, así como de otras variables demográficas y económicas que permiten profundizar en el análisis de los aspectos laborales (INEGI 2011).

Las bases de datos se homogeneizaron a partir de la ENOE en 2005, por lo que el alcance temporal de este documento se ubica a partir de dicho año de referencia y se toma como año de comparación el 2014. Cabe mencionar que se toman los datos del segundo trimestre de cada año para las 32 entidades federativas.

La muestra, representada por la población ocupada, arroja 167.378 casos para el segundo trimestre de 2005 y 169.365 para el mismo período de 2014. Al aplicar el factor de expansión, la población ocupada en México representó 41.193.204 personas $(96,5 \%$ del total de la PEA) y 49.822 .596 personas $(95,1 \%$ del total de la PEA), para 2005 y 2014 respectivamente (INEGI 2011).

\subsection{Operacionalización del concepto de flexibilidad laboral}

Como se mencionó en la sección teórica, la flexibilidad laboral se puede clasificar en cuatro tipos: flexibilidad interna, externa, salarial y extrema (Palacio y Álvarez 2004). De acuerdo con esta división se identificaron siete indicadores que representan la tipología de la flexibilidad laboral estatal. Los indicadores fueron:

a. Flexibilidad interna: porcentaje de población ocupada que tiene una jornada de tiempo parcial y porcentaje de población ocupada que tiene una jornada de tiempo extra.

b. Flexibilidad externa: porcentaje de población ocupada que trabaja por cuenta propia.

c. Flexibilidad salarial: porcentaje de población ocupada que recibe menos de dos salarios mínimos;

d. Flexibilidad extrema: porcentaje de población ocupada sin acceso a instituciones de salud, porcentaje de población ocupada que no tiene prestaciones laborales y porcentaje de población ocupada que trabaja en el sector informal.

Estos indicadores permiten analizar la diversidad de condiciones de trabajo de la población ocupada y clasificar, de esta manera, al empleo en flexible y no flexible. 


\subsection{Estimación y estratificación del índice de flexibilidad laboral}

A partir de los siete indicadores que caracterizaron la flexibilidad del trabajo se estimó el índice de flexibilidad laboral estatal a través del análisis de factores. Este último permite identificar los componentes que explican las correlaciones entre un conjunto de variables, y con ello describir el máximo de información contenida en los datos. Es importante señalar que se utilizó el método de "componentes principales", a través del paquete estadístico SPSS versión 21. Cabe señalar que los indicadores se estandarizaron para evitar que el análisis factorial diera mayor peso a las variables que tienen una mayor varianza. La factibilidad de los modelos se probó utilizando el estadístico Kaiser-Meyer-Olkin (KMO) y el test de Bartlett ${ }^{4}$.

Con base en este análisis se encontraron dos factores que se forman a partir de la alta correlación entre las variables. El primero está compuesto por seis indicadores (representados en porcentajes): población ocupada que tiene jornada de tiempo parcial, trabaja por cuenta propia, sin acceso a instituciones de salud, reciben menos de dos salarios mínimos, no tienen prestaciones laborales y trabajan en el sector informal. Estos se agrupan en un único factor que constituye un grupo diferenciado de variables dentro de la matriz de correlaciones. Este factor parece reflejar la dimensión de "condiciones de trabajo".

El segundo factor está formado por el indicador de "porcentaje de población ocupada que tiene una jornada de tiempo extra", lo que podría representar "el tiempo laboral excesivo". Estos resultados muestran un importante aspecto: que la flexibilidad laboral responde a un concepto multidimensional, en este caso dos dimensiones.

Las puntuaciones factoriales de la población ocupada llevaron a estimar el índice factorial de flexibilidad laboral, combinando cada factor con la variabilidad explicada por cada uno de éstos. En otras palabras, se multiplica cada uno de los factores obtenidos por el ponderador de cada uno de éstos y al final se suman estos productos.

Matemáticamente el índice se estimó de la siguiente manera:

$$
\stackrel{t}{t}=\sum_{i=1}^{n} C_{i} Z_{i}=C_{1} * Z_{1}+C_{2} * Z_{2}+\ldots+C_{n} * Z_{n}
$$

Donde:

IFL = índice de flexibilidad laboral

$t=$ año del índice

$\mathrm{C}_{i}=$ ponderador del factor $i$

$\mathrm{Z}_{i}=$ factor $i$ del análisis factorial

$n=$ número de factores

4 El resumen de la prueba KMO, con un nivel de significancia de 0,05, fue de 0,75 y 0,77 para 2005 y 2014 respectivamente; y para el caso de la prueba de Bartlett, los resultados, para ambos años, arrojaron un nivel crítico (sig.) igual a 0,000. Estas cifras indican que es factible realizar el análisis de factores con las variables propuestas (ver anexo). 
Una vez obtenido el índice de flexibilidad laboral, se realizó su estratificación a través de la técnica de Dalenius y Hodges (1959). Este método consiste en hallar una estratificación óptima, en la cual la varianza del estimador sea mínima al interior de los grupos (grados), y al mismo tiempo la varianza del estimador sea máxima entre los grupos; en otras palabras, lograr que los grupos sean lo más homogéneos posible en su interior y lo más heterogéneos posible y diferenciables entre sí. La variable resultante de estratificación tiene la característica de ser continua y tener una distribución aproximadamente normal garantizando la partición óptima de estratos.

Al aplicar la técnica de Dalenius y Hodges (1959) se estratificó el índice de flexibilidad laboral y se encontraron tres estratos o grados (h) para las 32 entidades (n) (INEGI 2010). Para ello se siguieron los sucesivos pasos:

1. Se ordenaron las observaciones de manera ascendente.

2. Se agruparon en $J$ clases, donde $J=\min \{h * 10, n\}$.

3. Los límites de cada clase se estimaron de los siguiente manera:

$$
\begin{array}{ll}
\lim \inf C_{k}=\min \left(x_{i}\right)+(k-1) * \frac{\max \left(x_{i}\right)-\min \left(x_{i}\right)}{J} & \text { (Ecuación 2) } \\
\lim \sup C_{k}=\min \left(x_{i}\right)+(k) * \frac{\max \left(x_{i}\right)-\min \left(x_{i}\right)}{J} & \text { (Ecuación 3) }
\end{array}
$$

Los intervalos se tomaron abiertos por la izquierda y cerrados por la derecha, a excepción del primero que fue cerrado por ambos lados.

4. A partir de estos límites, se obtuvo la frecuencia de observaciones en cada clase:

$$
f_{i} i \in\{1, \ldots, J\}
$$

(Ecuación 4)

5. Se calculó la raíz cuadrada de las frecuencias de cada clase.

6. Se acumuló la raíz cuadrada de las frecuencias de cada clase:

$$
\sum_{1=1}^{J} \sqrt{f_{i}}
$$

(Ecuación 5)

7. Se dividió la suma de la raíz cuadrada de las frecuencias por el número de estratos:

$$
Q=\frac{1}{h} \Sigma_{i=1}^{J} \sqrt{f_{i}}
$$

(Ecuación 6)

8. Los puntos de corte de cada estrato se tomaron sobre el acumulado de la raíz cuadrada de las frecuencias de cada clase de acuerdo con el siguiente criterio: Q,22,...,(h-1)Q. Cuando el valor de Q quedó entre dos clases, se tomó como punto de corte aquella clase que presentaba la mínima distancia a Q. 
Los límites de los $h$ estratos conformados fueron aquellos correspondientes a los límites inferior y superior de las clases correspondientes en cada estrato.

La aplicación de esta técnica llevó a dividir en tres estratos el rango del índice de flexibilidad laboral. Por lo tanto, la estratificación del índice ayudó a segmentar la población ocupada según sus condiciones de trabajo. Esto permite identificar a las entidades con un nivel de flexibilidad laboral bajo, medio y alto. El cuadro 1 muestra el límite inferior y superior así como el número de entidades que se encuentran en cada nivel de flexibilidad laboral para los años 2005 y 2014.

Cuadro 1. Límite inferior y superior de los niveles de flexibilidad laboral y número de entidades según nivel

\begin{tabular}{lcccccc} 
Nivel & \multicolumn{3}{c}{$\mathbf{2 0 0 5}$} & \multicolumn{3}{c}{$\mathbf{2 0 1 4}$} \\
\cline { 2 - 4 } & $\begin{array}{c}\text { Límite } \\
\text { inferior }\end{array}$ & $\begin{array}{c}\text { Límite } \\
\text { superior }\end{array}$ & Entidades & $\begin{array}{c}\text { Límite } \\
\text { inferior }\end{array}$ & $\begin{array}{c}\text { Límite } \\
\text { superior }\end{array}$ & Entidades \\
\hline Bajo & $-1,18$ & $-0,26$ & 11 & $-1,35$ & $-0,38$ & 11 \\
Medio & $-0,26$ & 0,40 & 11 & $-0,38$ & 0,24 & 11 \\
Alto & 0,40 & 1,32 & 10 & 0,24 & 1,30 & 10 \\
\hline
\end{tabular}

Fuente: elaboración propia con base en INEGI (2005), INEGI (2014). Segundo trimestre.

Para la validez de los tres niveles de flexibilidad laboral se llevó a cabo el análisis de consistencia interna y externa. Para la consistencia interna se realizó la comparación de los siete indicadores incluidos en la estimación del índice de flexibilidad con el valor obtenido de éste. Por su parte, la consistencia externa se analizó contrastando los tres niveles de flexibilidad laboral contra una variable externa. En este caso se eligió la variable tamaño de establecimiento. La conclusión del análisis de consistencia interna y externa sobre los tres niveles de flexibilidad laboral permite sugerir que los resultados obtenidos son confiables. Esto se debe a que no se presentó ningún patrón anormal que pusiera en duda la consistencia de la agrupación obtenida. En suma, la diversidad de situaciones laborales permitió segmentar la población ocupada de cada entidad federativa en tres grados de flexibilidad laboral de acuerdo con sus condiciones laborales.

\section{Niveles de flexibilidad laboral en México y en sus entidades}

El nivel bajo de flexibilidad laboral se caracteriza por que entre $17 \%$ y $57 \%$ de la población ocupada se ve afectada en sus condiciones de trabajo. Este nivel de flexibilidad laboral representa el menor grado de deterioro. Por ello constituye la mejor categoría donde las entidades podrían ubicarse. Las características de este nivel de flexibilidad coinciden con el mercado primario por ser empleos más atractivos en la lógica de la TSMT. 
El nivel medio de flexibilidad laboral se caracteriza por que entre $22 \%$ y $63 \%$ de la población ocupada tiene malas condiciones de trabajo. Comparado con el nivel bajo es posible observar una mayor proporción de población ocupada que se encuentra desarrollando actividades laborales flexibles.

Por su parte, el nivel alto de flexibilidad laboral se distingue por un mayor porcentaje de población ocupada que tiene malas condiciones laborales: la proporción varía entre $30 \%$ y $76 \%$. En este nivel de flexibilidad laboral los empleos son los más deteriorados y por lo tanto los menos atractivos. Las características de este nivel de flexibilidad laboral se acercan más al mercado secundario bajo la TSMT. Los mapas 1 y 2 muestran la estratificación de las entidades federativas según nivel de flexibilidad laboral.

Como se puede observar, en el 2005 las entidades del norte de México se ubican en el nivel de flexibilidad bajo. También sobresalen dos entidades del centro (Aguascalientes y Querétaro), una del Pacífico (Colima) y dos del sureste (Tabasco y Quintana Roo) que se ubican en este mismo nivel. Por su parte, las entidades del centro y sur de México destacan por situarse en el nivel alto de flexibilidad, a excepción del Estado de México y distrito federal, los cuales se ubican en el nivel medio. También aquellas entidades que tienen colindancia con las entidades del norte se ubican en el nivel medio de flexibilidad laboral. En el 2014 se observa un panorama muy similar, con algunos cambios importantes a destacar. Por ejemplo: Sinaloa y Jalisco se ubicaron en una mejor situación de la que se encontraban en el 2005; mientras que en Colima y Tabasco ocurrió lo contrario (cuadro 2).

Mapa 1. Niveles de flexibilidad laboral en México, 2005

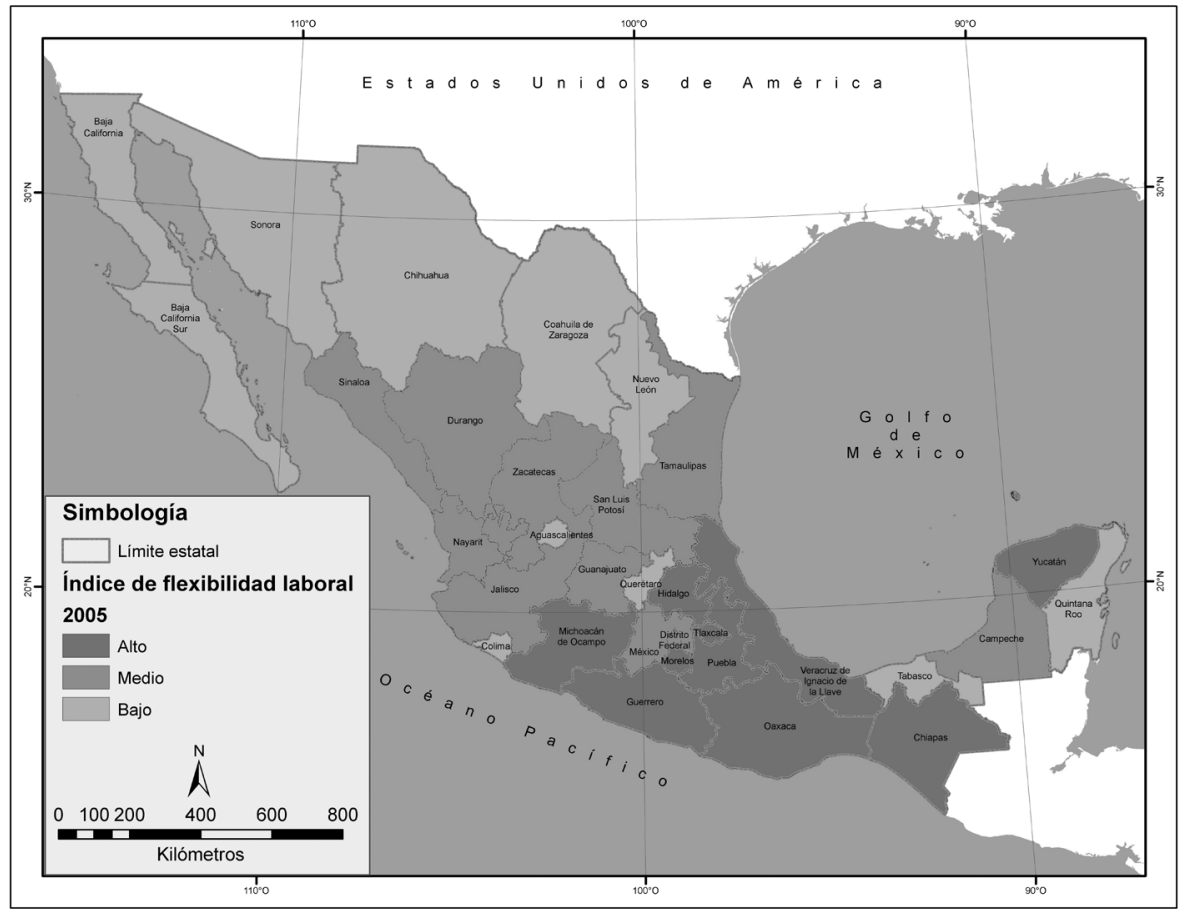

Fuente: elaboración propia. 
Mapa 2. Niveles de flexibilidad laboral en México, 2014

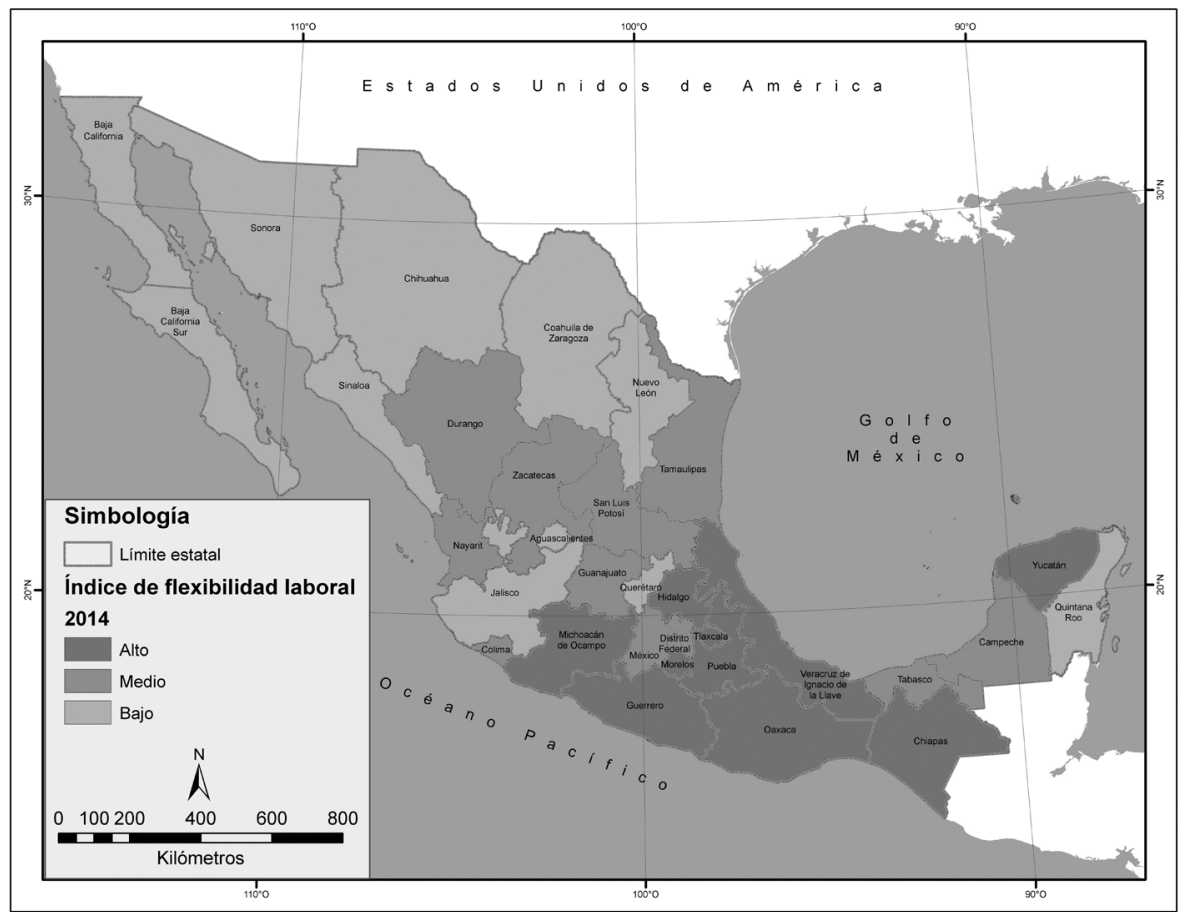

Fuente: elaboración propia.

Cuadro 2. Índice y nivel de flexibilidad laboral según entidades de México, 2005 y 2014

\begin{tabular}{lrrrr}
\hline \multirow{2}{*}{ Entidad } & \multicolumn{2}{c}{ 2005 } & \multicolumn{2}{c}{ 2014 } \\
\cline { 2 - 5 } & \multicolumn{1}{c}{ Índice } & Nivel & Índice & Nivel \\
\hline Aguascalientes & $-0,4248$ & Bajo & $-0,4153$ & Bajo \\
Baja California & $-0,8582$ & Bajo & $-0,7884$ & Bajo \\
Baja California Sur & $-0,8418$ & Bajo & $-0,7569$ & Bajo \\
Campeche & 0,3641 & Medio & 0,1506 & Medio \\
Chiapas & 0,7784 & Alto & 0,7434 & Alto \\
Chihuahua & $-1,1785$ & Bajo & $-1,3545$ & Bajo \\
Coahuila & $-0,8653$ & Bajo & $-1,0457$ & Bajo \\
Colima & $-0,3772$ & Bajo & $-0,2012$ & Medio \\
Distrito Federal & $-0,2257$ & Medio & 0,1600 & Medio \\
Durango & $-0,2183$ & Medio & $-0,1355$ & Medio \\
Estado de México & 0,1416 & Medio & 0,1952 & Medio \\
Guanajuato & $-0,1917$ & Medio & 0,0112 & Medio \\
Guerrero & 0,9292 & Alto & 1,2142 & Alto \\
Hidalgo & 0,5137 & Alto & 0,4038 & Alto
\end{tabular}


Cuadro 2. Índice y nivel de flexibilidad laboral según entidades de México, 2005 y 2014

\begin{tabular}{lrrrr}
\hline Jalisco & $-0,1188$ & Medio & $-0,4711$ & Bajo \\
Michoacan & 0,5536 & Alto & 0,5423 & Alto \\
Morelos & 0,5198 & Alto & 0,6685 & Alto \\
Nayarit & 0,3493 & Medio & 0,2362 & Medio \\
Nuevo León & $-1,0168$ & Bajo & $-0,9302$ & Bajo \\
Oaxaca & 1,3177 & Alto & 1,1637 & Alto \\
Puebla & 0,6660 & Alto & 0,6802 & Alto \\
Querétaro & $-0,5367$ & Bajo & $-0,7441$ & Bajo \\
Quintana Roo & $-0,3669$ & Bajo & $-0,5323$ & Bajo \\
San Luís Potosí & 0,2015 & Medio & 0,0716 & Medio \\
Sinaloa & $-0,0785$ & Medio & $-0,5333$ & Bajo \\
Sonora & $-0,4783$ & Bajo & $-0,6938$ & Bajo \\
Tabasco & $-0,2934$ & Bajo & $-0,0483$ & Medio \\
Tamaulipas & $-0,1329$ & Medio & $-0,0164$ & Medio \\
Tlaxcala & 1,1742 & Alto & 1,3036 & Alto \\
Veracruz & 0,4346 & Alto & 0,4324 & Alto \\
Yucatán & 0,5639 & Alto & 0,6882 & Alto \\
\hline Zacatecas & $-0,0039$ & Medio & 0,0020 & Medio
\end{tabular}

Fuente: elaboración propia con base en INEGI (2005) e INEGI (2014).

Así, la evidencia empírica muestra la existencia de la diversidad de situaciones de flexibilidad laboral en las que se puede hallar la población ocupada. Como consecuencia de los cambios en el modelo económico y en el sistema productivo, aunados a las modificaciones legales en la reforma laboral mexicana -o incluso sin éstas-, han impuesto un nuevo contexto laboral, caracterizado por relaciones de trabajo donde impera la flexibilidad de las condiciones laborales de los ocupados, aspectos que promueven situaciones de empleo flexibles o también denominadas formas particulares de empleo, tal como lo denomina Neffa (2008). En este contexto, se visualiza la hipótesis básica de la flexibilidad laboral, la cual plantea la eliminación y/o la reducción sobre la intervención de las instituciones en los cambios, mecanismos, formas de contratación y uso intensivo de la población ocupada.

En la configuración de este nuevo ámbito laboral, las estrategias empresariales tienen un papel primordial, puesto que establecen la flexibilidad de jornadas de trabajo, de contratación, de salario, entre otras. Estas formas de flexibilidad laboral conducen, a su vez, a la desigualdad entre las regiones, entidades o ciudades de México, como se ha evidenciado en otros estudios (Román y Cervantes 2013). Se puede observar que las entidades cercanas a Estados Unidos de América ofrecen mejores condiciones de empleo debido a que presentan una estructura económica más consolidada, en comparación con entidades localizadas en el centro y sur de México, donde se ofrecen empleos menos atractivos. 
De manera que se encuentra evidencia de la flexibilidad y segmentación laboral que experimenta la población ocupada mexicana. A partir de ahí se puede concluir la presencia de un mercado laboral flexible en México para los dos años analizados (2005 y 2014), aunque con ciertos matices y diferente intensidad en el país.

Con sano criterio, los resultados conducen a pensar en una correspondencia entre la evidencia empírica y el sustento teórico, y con ello, en la existencia de múltiples mercados de trabajo. De manera más clara se puede percibir un mercado primario -mejor estructurado con empleos más atractivos- ara las entidades del norte de México y un mercado secundario -con empleos más flexibles- para las entidades del centro y sur del país. Por lo tanto, la teoría de mercados segmentados ha sido fundamental para entender los diferentes grados de flexibilidad y ubicar las condiciones laborales de la población ocupada en diferentes niveles de deterioro.

Habría que decir también que la oferta de empleos más atractivos -menos flexibles- en las entidades del norte de México podría estar sustentada en la estructura económica de éstas. Al respecto, se debe reconocer que las entidades fronterizas, caracterizadas por la presencia de la industria maquiladora, mantienen una relación económica más consolidada, principalmente, con Estados Unidos de América. Asimismo, los estados del norte, se vinculan con centros y cadenas globales de producción relacionados con la electrónica. Actividad económica que se identifica por ser una de las más dinámicas, globalizadas y especializadas, por lo que las empresas enlazadas con esta industria, se han aglomerado constituyendo verdaderos clusters con derramas importantes a la economía de la región (Román y Cervantes 2013).

Por su parte, las entidades del centro se caracterizan por tener un sector industrial relativamente fuerte, especialmente en el sector automotor, de alimentos, de bebidas y tabaco, así como la industria química. Estos sectores económicos son importantes pero menos dinámicos que el ramo electrónico que se registra en las entidades del norte. Este elemento aunado a una estructura económica donde no prevalecen los grandes clústeres industriales especializados -por el contrario en estas entidades destacan los micronegocios, pequeños y medianos establecimientos- hacen que una mayor cantidad de población ocupada se encuentre en empleos con condiciones laborales más flexibles.

Llaman la atención los casos de Quintana Roo (ubicado en la Riviera Maya) y Querétaro (en el centro del país), debido a que estas entidades no colindan con los Estados Unidos de América pero se ubican en un nivel de flexibilidad bajo. La primera entidad se caracteriza por tener una estructura económica donde sobresale el sector terciario, en especial el turístico, con una representación importante. Mientras en Querétaro destaca el sector industrial consolidado. De modo que las mejores condiciones laborales que ofrecen estas entidades se podrían sustentar, en parte, por el crecimiento del sector turístico así como del sector industrial. Aunque se ha evidenciado en otros estudios (Román y Cervantes 2013) que en el turismo es donde prevalecen las condiciones laborales más precarias; no obstante, los datos de esta investigación no aportan evidencias para sostener y apoyar este argumento. 
En suma, la presencia de empleos más o menos flexibles en México se centra principalmente en la existencia de estructuras económicas diferentes, las cuales prevalecen en cada entidad, aunado a la importancia que tiene cada sector económico, el papel de las organizaciones, la presencia o ausencia de clústeres y, en general a la dinámica de la economía que predomina en cada entidad.

Una vez explicados y discutidos los resultados, es preciso establecer las limitantes que de alguna manera abren la oportunidad para conducir futuras investigaciones. El índice se estimó considerando todas las actividades económicas de manera conjunta. Sería óptimo lograr diferenciar este indicador en los tres sectores económicos principales, ya que es bien sabido que en la frontera norte la existencia de la industria maquiladora ha precarizado el nivel salarial de los trabajadores, por lo que desglosar las actividades permitiría un mayor nivel de análisis. Asimismo, sería bueno realizar el estudio a escala regional o de entidades que permitan conjuntar los resultados con un estudio sobre la estructura económica, por lo que habría que bajar el nivel de información a los municipios realizando así mayores aportaciones a los estudios espaciales, es decir, a escala local.

\section{Conclusiones}

A partir de los resultados obtenidos en esta investigación se pueden realizar las siguientes conclusiones:

- La flexibilidad del mercado de trabajo es resultado de la reestructuración económica y productiva para mejorar los niveles de productividad y beneficio.

- El índice de flexibilidad laboral estimado permite identificar las diferencias en el grado de flexibilización espacial.

- Existen desigualdades territoriales con un patrón definido: el norte se caracteriza por ser la región con menos grado de flexibilidad laboral, es decir, jornadas laborales que respetan las ocho horas de ley, empleados que pertenecen a una empresa de carácter formal y no lo hacen por cuenta propia, trabajadores con acceso a instituciones de salud, ingreso superior a los dos salarios mínimos, así como prestaciones laborales. Los estados del sur y del centro, a excepción del distrito federal y el Estado de México, presentan una situación diferente con altos niveles de flexibilidad laboral; las entidades de la parte baja del norte, junto con la capital del país y la entidad mexiquense, se ubican con un nivel de flexibilidad laboral medio.

- $\mathrm{Al}$ observarse disimilitudes espaciales se confirma la existencia de más de un mercado de trabajo y por lo tanto de diferencias laborales en el territorio mexicano.

- La temporalidad en el índice de flexibilidad (2005 y 2014) posibilita establecer la existencia de formas de trabajo flexibles previas a la reforma laboral realizada en México.

El hallazgo de desigualdades territoriales coincide con lo señalado por Alba (2007) y Asuad, Quintana y Ramírez (2007) quienes ya habían detectado en investigaciones anteriores la existencia de diferencias regionales, pero no en función de la flexibilidad laboral. 
Las desigualdades espaciales en México indican que los cambios adoptados por empresarios, a partir de la globalización económica y la reestructuración productiva, han ayudado a la presencia de la flexibilización laboral en todo el territorio, aunque con diferente intensidad dentro de éste, a partir de a) la puesta en marcha del outsorcing o externalización de la mano de obra, b) la desvinculación entre obrero-patrón, y c) el debilitamiento del papel de las instituciones para defender los derechos laborales ganados con anterioridad.

Con lo anterior se confirma que la segmentación de los mercados de trabajo en México, tal como lo establece la TSMT, se presentó en todo el territorio nacional en 2005 y 2014. De manera específica sobresalen los empleos menos atractivos, los cuales se ubican en el segmento secundario contra los empleos menos flexibles que se encuentran en el sector primario y tienen menor presencia. Por lo tanto, el intento para probar la hipótesis planteada sobre las diferencias espaciales sugiere ser aceptada, de ahí que este documento sirve como base para los estudios de la TSMT que puedan aportar avances en el ámbito espacial, así como a la cuarta generación de esta teoría como lo establece Peck (1996).

Finalmente, todo parece confirmar que la presencia de la flexibilidad de las condiciones de trabajo se presentó con anterioridad a la reforma laboral realizada el 29 de septiembre de 2012, de manera que en este nuevo modelo económico la flexibilidad laboral es una las características y patrones que describen de mejor forma el nuevo ámbito de trabajo en México y en sus entidades y que se formalizó a partir de los cambios aprobados en la Ley del trabajo (Román y Cervantes 2013). Por lo tanto, este documento aporta más evidencia sobre la presencia de la flexibilidad laboral en México desde el 2005, cuando una parte importante de los trabajadores ya tenían condiciones laborales flexibles antes de que la reforma laboral se formalizara.

\section{Referencias bibliográficas}

Alba, Carlos. «Globalización y desarrollo regional en México». En Agenda para el Desarrollo. Políticas de Desarrollo Regional Vol. 13, de José Calva (Coord.), 34-55. México D.F.: Porrúa, Cámara de Diputados e IIEc, 2007.

Antunes, Ricardo. Los Sentidos del Trabajo. Buenos Aires: Ediciones Herramientas y Taller de Estudios Laborales, 2005.

Asuad, Normand, Luis Quintana y Roberto Ramírez. «Desarrollo y políticas regionales en México: retos y perspectivas 2006-2020». En Agenda para el Desarrollo. Políticas de Desarrollo Regional Vol. 13, de José Calva (Coord.), 231-263. México D.F.: Porrúa, Cámara de Diputados e IIEc, 2007.

D'Addio, Anna, and Michael Rosholm. «Temporary Employment in Europe: characteristics, Determinants and Outcomes». Brussels Economic Review, Vol. 48, $\mathrm{n}^{\circ} 1 / 2$ (Summer 2005): 13-42.

Dalenius, Tore, and Joseph Hodges. «Minimum Variance Stratification». Journal of the American Statistical Association, Vol. 54, nº 285 (March 1959): 88-101.

De la Garza, Enrique. Reestructuración Productiva, empresas y trabajadores en México. México D.F.: UAM y FCE, 2006.

De Oliveira, Orlandina. "Jóvenes y precariedad laboral en México». Papeles de Población, Vol. 12, nº 49 (julio-septiembre 2006): 37-73. 
DOF (Diario Oficial de la Federación). Decreto por el que se reforman, adicionan y derogan diversas disposiciones de la Ley Federal del Trabajo, 2012 Disponible en http://www.dof.gob.mx/copias_cert.php?acc=ajaxPaginas\&paginas=1-120\&se ccion=PRIMERA\&edicion=249554\&ed=MATUTINO\&fecha=30/11/2012 (último acceso: 11 de diciembre de 2015).

Edwards, Paul. «Non-standard Work and Labour Market Re-structuring in the UK». Paper for Associazione Nuovi Lavori conference on The Latest in the Labour Market, Rome, February 2006.

European Commission. Eurostat, 2015. http://ec.europa.eu/eurostat/data/ database (último acceso: 26 de mayo de 2015).

Ferreiro, Jesús. «Políticas de rentas y reformas laborales en España». Revista del Ministerio de Trabajo y Asuntos Sociales, $\mathrm{n}^{\circ} 46$ (2003): 15-40.

García, Beatriz, Edmar Salinas, Leticia Velázquez, Zorayda Carranco y Andrés Godínez. "Lo cotidiano del sector industrial en México: 25 años de cambio estructural». El Cotidiano, n ${ }^{\circ} 156$ (julio-agosto 2009): 77-107.

García, Brígida. «Reestructuración económica y feminización del mercado de trabajo en México». Papeles de Población, Vol. 7, n 27 (enero-marzo 2001): 45-61.

Gittleman, Maury, and David Howell. "Changes in the Structure and Quality of Jobs in the United States: Effects by Race and Gender, 1973-1990». Industrial and Labor Relations Review, Vol. 48, $\mathrm{n}^{\circ} 3$ (April 1995): 420-440.

Gutiérrez, Esthela. «Nuevos escenarios en el mercado de trabajo en México, 19831998». Papeles de Población, Vol. 5, nº 21 (julio-septiembre 1999): 21-55.

Harvey, David. La condición de la posmodernidad. Investigación sobre los orígenes del cambio cultural. Buenos Aires: Amorrortu editores, 1998.

Infante, Ricardo y Emilio Klein. «Mercado latinoamericano del trabajo en 19501990». Revista de la CEPAL, $\mathrm{n}^{\circ} 45$ (diciembre 1991): 129-144.

INEGI (Instituto Nacional de Estadística y Geografía). ¿Cómo se hace la ENOE: método y procedimientos?, 2011. http://www.inegi.org.mx/est/contenidos/espanol/ metodologias/enoe/ENOE_como_se_hace_la_ENOE1.pdf (último acceso: 12 de septiembre de 2015).

INEGI. Encuesta Nacional de Ocupación y Empleo (ENOE). México D.F.: INEGI, 2005.

INEGI. Encuesta Nacional de Ocupación y Empleo (ENOE). México D.F.: INEGI, 2014.

INEGI. "Nota técnica. Estratificación univariada". Censo de Población y Vivienda, 2010. SINCE. Sistema para la Consulta de Información Censal. México D.F.: INEGI, 2010.

Lóyzaga, Octavio. «Los grandes retrocesos de la reforma laboral». El Cotidiano, $\mathrm{n}^{\circ}$ 178 (marzo-abril 2013): 45-51.

Mora, Minor. Ajuste y empleo La precarización del trabajo asalariado en la era de la globalización. México D.F.: El Colegio de México, 2010.

Neffa, Julio. «Las teorías de la segmentación de los mercados de trabajo». En Teorías económicas sobre el mercado de trabajo. III Análisis institucionalistas, de François Eymard-Duvernay y Julio Neffa, 139-206. Buenos Aires: FCE, 2008.

Palacio, Juan y Carlos Álvarez. El mercado de trabajo: análisis y políticas. Madrid: Ediciones Akal, 2004. 
Peck, Jamie. Work-Place. The Social Regulation of Labor Markets. New York: The Guildford Press, 1996.

Piore, Michael «Dualism in the Labor Market: A Response to Uncertainty and Flux. The Case of France». Revue Économique, Vol. 29, nº 1 (janvier 1978): 26-48.

Reich, Michael, David Gordon and Richard Edwards. «Dual Labor Markets: A Theory of Labor Market Segmentation». American Economic Review, Vol. 63, $\mathrm{n}^{\circ} 2$ (May 1973): 359-365.

Reynolds, Jeremy, and Jeffrey Wenger. «Prelude to a RIF: Older Workers, Part-Time Hours, and Unemployment». Journal of Aging and Social Policy, Vol. 22, $\mathrm{n}^{\circ}$ 2 (April 2010): 99-116.

Rodríguez, Martín, Analía Erbes, Sonia Roitter y Andrea Pujol. «El nuevo trabajo industrial: una mirada crítica desde la calidad del empleo». Sociedad y economía, nº 28 (enero-junio 2015): 137-162.

Román, Yuliana y David Cervantes. «El empleo precario de jóvenes asalariados en México. El caso de Toluca, Tijuana y Mérida (2005-2010)». Revista Facultad de Ciencias Económicas: Investigación y Reflexión, Vol. XXI, nº 1 (junio 2013): 43-74.

Tompson, William. The Political Economy of Reform, 2009. http://www.oecd.org/ site/sgemrh/46190166.pdf (último acceso: 10 de septiembre de 2015).

Vega, María. La Reforma Laboral en América Latina: 15 años después. Un análisis comparado. Lima: OIT, 2005.

Williamson, John. «No hay consenso. Reseña sobre el Consenso de Washington y sugerencias sobre los pasos a dar». Finanzas y Desarrollo (septiembre 2003). Disponible en http://www.imf.org/external/pubs/ft/fandd/spa/2003/o9/pdf/ williams.pdf (último acceso: 12 de septiembre de 2015).

\section{Anexo}

\section{Resultados del índice de flexibilidad laboral en México, 2005 y 2014}

Cuadro 1. Pruebas de KMO y prueba de Bartlett

\begin{tabular}{cccc}
\hline & $\mathbf{2 0 0 5}$ & $\mathbf{2 0 1 4}$ \\
\hline \multicolumn{2}{c}{$\begin{array}{c}\text { Medida de adecuación muestral de Kaiser- } \\
\text { Meyer-Olkin. }\end{array}$} &, 753 &, 775 \\
$\begin{array}{c}\text { Prueba de esfericidad } \\
\text { de Bartlett }\end{array}$ & $\begin{array}{c}\text { Chi-cuadrado } \\
\text { aproximado }\end{array}$ & 204,909 & 208,559 \\
& gl & 21 & 21 \\
& Sig. &, 000 &, 000 \\
\hline
\end{tabular}

Fuente: elaboración propia con base en análisis de factores 
Cuadro 2. Comunalidades

\begin{tabular}{lccccc}
\hline & \multicolumn{2}{c}{2005} & & \multicolumn{2}{c}{2014} \\
& Inicial & Extracción & Inicial & Extracción \\
\hline Tiempo_parcial & 1,000 &, 710 & 1,000 &, 766 \\
Tiempo_extra & 1,000 &, 918 & 1,000 &, 890 \\
Traba_ctap & 1,000 &, 645 & 1,000 &, 814 \\
Sin_acceso_inst & 1,000 &, 936 & 1,000 &, 938 \\
Ingreso_2sm & 1,000 &, 676 & 1,000 &, 647 \\
Sin_prestaciones & 1,000 &, 909 & 1,000 &, 915 \\
Sec_informal & 1,000 &, 695 & 1,000 &, 749 \\
\hline
\end{tabular}

Fuente: elaboración propia con base en análisis de factores

Cuadro 3. Varianza total explicada

\begin{tabular}{|c|c|c|c|c|c|c|c|c|c|}
\hline \multirow{3}{*}{ Componente } & \multicolumn{6}{|c|}{2005} & & & \\
\hline & \multicolumn{3}{|c|}{ Autovalores iniciales } & \multicolumn{3}{|c|}{$\begin{array}{l}\text { Sumas de las saturacio- } \\
\text { nes al cuadrado de la } \\
\text { extracción }\end{array}$} & \multicolumn{3}{|c|}{$\begin{array}{c}\text { Suma de las saturaciones } \\
\text { al cuadrado de la rota- } \\
\text { ción }\end{array}$} \\
\hline & Total & $\begin{array}{l}\% \text { de la } \\
\text { varian- } \\
\text { za }\end{array}$ & $\begin{array}{l}\% \\
\text { acumu- } \\
\text { lado }\end{array}$ & Total & $\begin{array}{l}\% \text { de la } \\
\text { varian- } \\
\quad \text { za }\end{array}$ & $\begin{array}{l}\% \\
\text { acumu- } \\
\text { lado }\end{array}$ & Total & $\begin{array}{l}\text { \% de la } \\
\text { varian- } \\
\text { za }\end{array}$ & $\begin{array}{c}\% \\
\text { acumu- } \\
\text { lado }\end{array}$ \\
\hline 1 & 4,432 & 63,320 & 63,320 & 4,432 & 63,320 & 63,320 & 4,256 & 60,806 & 60,806 \\
\hline 2 & 1,057 & 15,094 & 78,413 & 1,057 & 15,094 & 78,413 & 1,232 & 17,607 & 78,413 \\
\hline 3 & ,660 & 9,433 & 87,847 & & & & & & \\
\hline 4 & ,414 & 5,914 & 93,761 & & & & & & \\
\hline 5 & ,237 & 3,384 & 97,145 & & & & & & \\
\hline 6 & 189 & 2,696 & 99,842 & & & & & & \\
\hline 7 & ,011 & 158 & 100,000 & & & & & & \\
\hline \multicolumn{10}{|c|}{2014} \\
\hline 1 & 4,628 & 66,107 & 66,107 & 4,628 & 66,107 & 66,107 & 4,499 & 64,270 & 64,270 \\
\hline 2 & 1,092 & 15,597 & 81,704 & 1,092 & 15,597 & 81,704 & 1,220 & 17,435 & 81,704 \\
\hline 3 & , 444 & 6,346 & 88,050 & & & & & & \\
\hline 4 & ,401 & 5,722 & 93,772 & & & & & & \\
\hline 5 & 301 & 4,304 & 98,076 & & & & & & \\
\hline 6 & 117 & 1,673 & 99,749 & & & & & & \\
\hline 7 & ,018 & ,251 & 100,000 & & & & & & \\
\hline
\end{tabular}

Fuente: elaboración propia con base en análisis de factores. 
Cuadro 4. Matriz de componentes

\begin{tabular}{llccc}
\hline \multicolumn{1}{c}{ Indicador } & \multicolumn{2}{c}{2005} & \multicolumn{2}{c}{$\mathbf{2 0 1 4}$} \\
& $\mathbf{1}$ & $\mathbf{2}$ & $\mathbf{1}$ & $\mathbf{2}$ \\
\hline Tiempo_parcial &, 684 &,- 492 &, 681 &,- 549 \\
Tiempo_extra &, 364 &, 886 &, 358 &, 873 \\
Traba_ctap &, 795 &,- 112 &, 902 &,- 013 \\
Sin_acceso_inst &, 967 &, 011 &, 968 &,- 022 \\
Ingreso_2sm &, 822 &,- 001 &, 803 &,- 041 \\
Sin_prestaciones &, 953 &,- 011 &, 957 &,- 007 \\
Sec_informal &, 824 &, 126 &, 851 &, 159 \\
\hline
\end{tabular}

Fuente: elaboración propia con base en análisis de factores.

Cuadro 5. Matriz de componentes rotados

\begin{tabular}{lcccc}
\hline \multicolumn{1}{c}{ Indicador } & \multicolumn{2}{c}{2005} & \multicolumn{2}{c}{$\mathbf{2 0 1 4}$} \\
& \multicolumn{2}{c}{ Componente } & $\mathbf{1}$ & $\mathbf{2}$ \\
\hline Tiempo_parcial &, 778 &,- 323 &, 773 &,- 409 \\
Tiempo_extra &, 152 &, 946 &, 184 &, 925 \\
Traba_ctap &, 800 &, 072 &, 888 &, 159 \\
Sin_acceso_inst &, 939 &, 232 &, 955 &, 163 \\
Ingreso_2sm &, 801 &, 187 &, 796 &, 113 \\
Sin_prestaciones &, 931 &, 207 &, 940 &, 3175 \\
Sec_informal &, 773 &, 311 &, 805 &, 318 \\
\hline
\end{tabular}

Fuente: elaboración propia con base en análisis de factores. 